\title{
New integrated noise absorbing coating
}

\author{
Z. J. Ji, J. M. Wang, G. H. Liu \& J. Wang \\ China Building Materials Academy, China
}

\begin{abstract}
Nowadays, with growing concerns about our living environment in general, environmental pollution is becoming the most important problem that the world faces. One aspect of that pollution is of noise, and our research has been focusing on the problem for a long time resulting in a product which is now available in the form of a coating. This noise absorbing coating has some virtues. Firstly, it is as easy to paint as a normal water-based coating which will not result in organic pollution. Secondly, artistic effect is also a characteristic when the coating is applied. Thirdly, it has an extra function which can adjust environmental humidity to a comfortable level. Finally, the most important point is that this product has a price advantage compared to similar products.
\end{abstract}

Keywords: integrated, noise absorbing, water-based, humidity absorption.

\section{Introduction}

Nowadays, noise pollution has become a new focus around the world. It has not only influenced people's normal life, but also induced some deadly disease. Since noise problems have become much more serious, the demand for a better environment and comfortable life increases. Materials, which can absorb and reduce noise waves, are strongly desired. Accordingly, researchers have paid much attention to the sound absorbing features of varied granular or particle materials, especially porous materials. The porous materials, including mineral materials, fibres, foams and perforated plates, are widely used in architectural acoustic design owing to their sound absorption characteristics [1-7]. And, porous materials have been successfully applied in noise barriers walls and freeways. But, porous materials are not good at low noise frequency. Even more, porous materials are usually quite thick and made into plates for application $[8,9]$. 
Porous plates have good performance in noise absorbing at some frequencies for indoor environment; but most of them are placed as ceilings or walls which are skilfully installed and need more space. There are very few thin coating products, which have good noise absorption performance reported. This paper focuses on designing a noise absorbing coating for living space. It has dual benefits with good absorbing performance on noise and moisture. It especially has great performance in medium and high frequency noise absorbing and better performance in low-frequency noise absorbing. The functional parts of the coating are mostly expanded perlite and woody fibre [10]. The addition of woody fibre helped the new integrated noise absorbing coating achieve good performance.

\section{Experimental study and methods}

Perlite is a siliceous volcanic, and expands by 10-30 times its original volume when heated up. Expanded perlites are usually made into plates for thermal insulation and noise absorbing. Good results on its performance have been reported in Asia, and especially in Japan [9].

Expanded perlite, especially with an open pore structure, is widely studied in the field of noise absorbing materials. It is well known that open pore material on its own has a good performance at noise absorbing [11]. In this paper, different kinds of expanded perlite are studied, ranging from close pore to open pore.

There are four different expanded-perlite samples as experiment objects: 1\# incomplete-expanded perlite, 2\# open-pore expanded perlite, 3\# close-pore expanded perlite, and 4\# waterproof expanded-perlite. Sample 3\# is from Xinyang, Henan; the other three samples are from Langfang, Hebei. They have an average particle diameter of $1 \mathrm{~mm}$. According to the prescription design of the coating, expanded-perlite is added into the coating as a ratio of $25 \%-35 \%$ which is for making a thick water-based coating. Here, a thick coating is compared to normal water-based coatings for indoor and outdoor walls.

Coating samples are sprayed on cement fibreboard with a diameter of $10 \mathrm{~mm}$. The thickness of dried coating is around $3 \mathrm{~mm}-5 \mathrm{~mm}$. In this experiment, spraying is chosen because it is easy and convenient and an artistic look can be achieved. Fig. 1 is the surface topography of four different samples. As shown in Fig.1, it can be seen that samples $1 \#$ and $2 \#$ have a porous surface, but sample $3 \#$ and sample $4 \#$ do not have that phenomena.

\subsection{Noise absorption coefficient test of expanded-perlite samples}

\subsubsection{Conditions of noise absorption test}

Noise absorption coefficient measured with the tube method. The frequency range is from $200 \mathrm{~Hz}$ to $2000 \mathrm{~Hz}$.

The test was based on GBJ 88-85 Survey Standard of Noise Absorption Coefficient of Tube Method and Specific Acoustic Impedance, Chinese standard. The environment condition was temperature $24^{\circ} \mathrm{Cand}$ relative humidity $50 \%$. The tests were done by the Institute of Acoustic, Chinese Academy of Sciences. 


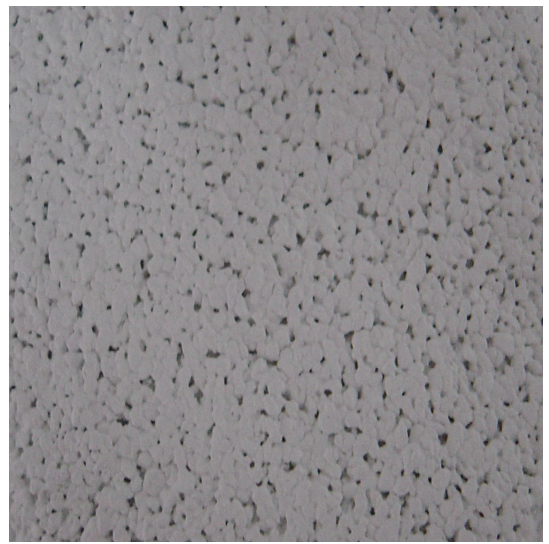

Sample 1\#

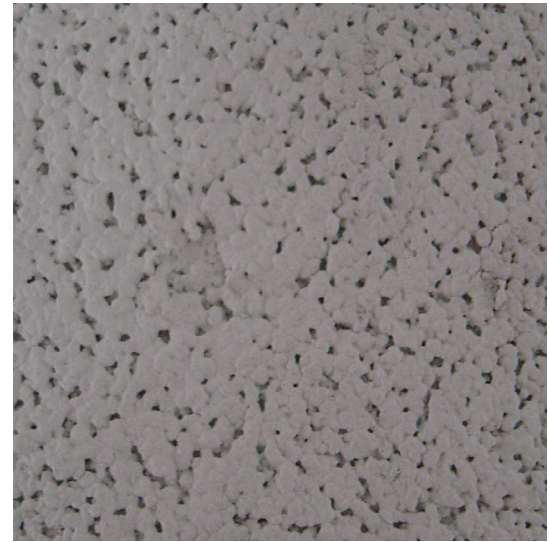

Sample 2\#

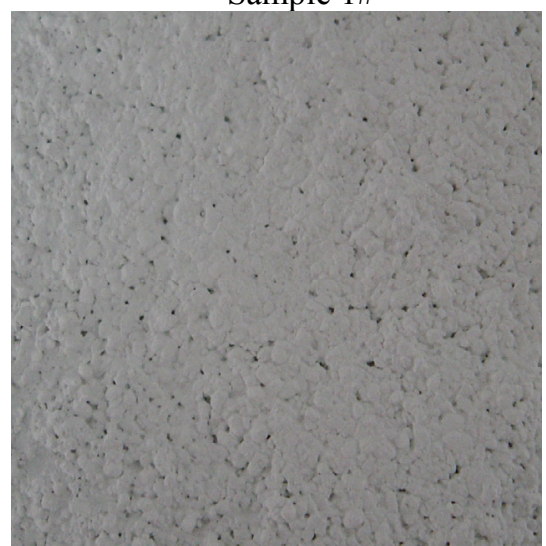

Sample 3\#

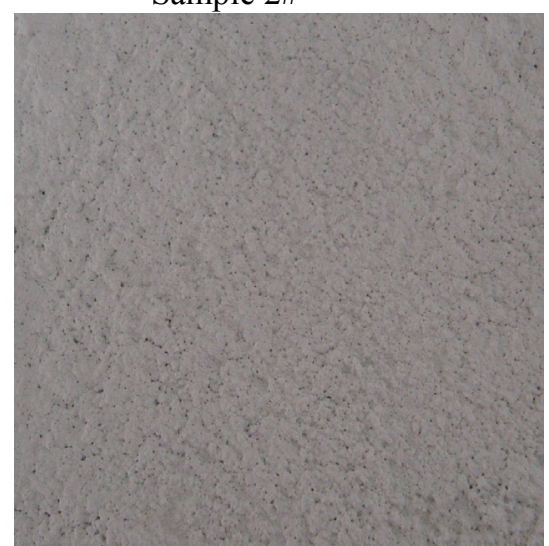

Sample 4\#

Figure 1: Examples of four expanded perlite coatings. 1\# is incompleteexpanded perlite coating. $2 \#$ is open-pore expanded perlite coating. $3 \#$ is close-pore expanded perlite coating. $4 \#$ is waterproof expanded-perlite coating.

Table 1: $\quad$ Testing machine.

\begin{tabular}{|c|c|}
\hline Name & Model/Standard \\
\hline Tube equipment & B\&K 4002 \\
\hline Multichannel analyzer & B\&KPULSE 3560 \\
\hline Power amplifier & B\&K 2706 \\
\hline Audio analyzer & B\&K 2112 \\
\hline
\end{tabular}

\subsubsection{Noise absorption coefficient results of four samples}

As shown in Fig.2, samples 1\# and 4\# have almost no performance in noise absorbing, no matter whether low or high frequency. Same as $1 \#$ and 4\#, samples 


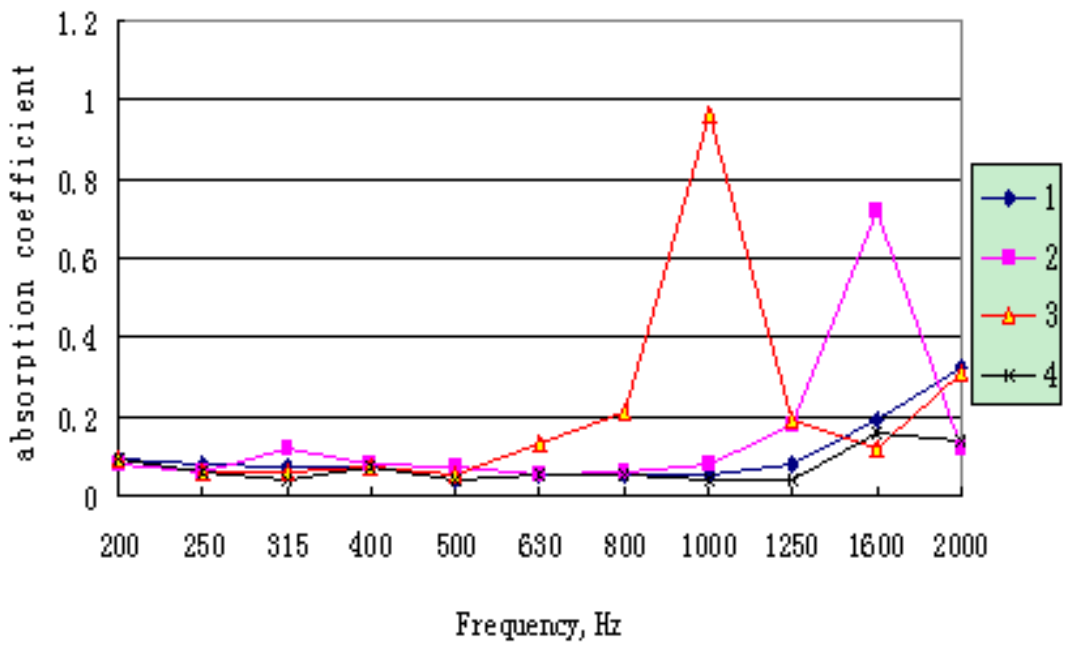

Figure 2: Experiment of noise absorption coefficient of expanded-perlite samples.

2\# and 3\# also are not good at low-frequency noise absorption; however, they have showed a strong ability of noise absorbing in medium- and high-frequency. Sample 2\#, added open-pore perlite, has a great performance around $1600 \mathrm{HZ}$. Sample 3\#, added close-pore perlite has a great performance around $1000 \mathrm{HZ}$. So it can be concluded that sample $2 \#$ and sample $3 \#$, namely open and close pore perlite, have good noise absorption.

According to existing research, close-pore perlite is widely considered as a poor material in the noise-absorbing process. But in this experiment, close-pore perlite shows a wonderful character in reducing noise.

Normally, people are very sensitive about high-frequency noise and relatively insensitive about low-frequency noise. However, people are always getting troubled by low-frequency noise and our coating belongs to indoor paints. Therefore, further study will be focused on improving performance in lowfrequency noise reduction.

\subsection{Humidity adjusting test of expanded-perlite samples}

\subsubsection{Conditions of humidity adjusting test}

The test was based on Chinese industry standard of building materials, Testing Methods of Moisture Absorption and Desorption of Building Materials. A humidity absorption experiment of four samples was processed under relative humidity $95 \%$ within 24 hours; followed by a 24-hour process of humidity desorption experiment under relative humidity $30 \%$. The results are figured out according to the standard. 


\subsubsection{Humidity adjusting results of four samples}

The result in table 2 show two sets of data: the first is humidity absorption and desorption per unit area; the second is humidity absorption and desorption per unit mass. Because unavoidable errors in the painting process, the ranges of the two sets of data may not be matched perfectly. Combining the whole data, it shows a strong humidity absorbing ability of samples $1 \#, 2 \#$, and 3\#. Comparing with humidity absorption ability, humidity desorbing performance is more important.

Table 2: Humidity adjusting test results.

\begin{tabular}{|c|c|c|c|c|}
\hline Performance & $1 \#$ & $2 \#$ & $3 \#$ & $4 \#$ \\
\hline Humidity absorption $\left(\mathrm{g} / \mathrm{m}^{2}\right)$ & 226.24 & 223.84 & 251.36 & 188 \\
\hline Humidity desorption $\left(\mathrm{g} / \mathrm{m}^{2}\right)$ & 161.92 & 208.8 & 197.92 & 124.32 \\
\hline $\begin{array}{c}\text { Effect of Humidity } \\
\text { absorption } \times 1000(\mathrm{~g} / \mathrm{g})\end{array}$ & 53 & 49 & 44 & 42 \\
\hline $\begin{array}{c}\text { Effect of Humidity } \\
\text { desorption } \times 1000(\mathrm{~g} / \mathrm{g})\end{array}$ & 38 & 46 & 34 & 28 \\
\hline
\end{tabular}

Consequently, samples $2 \#$ and $3 \#$ are considered as the best two humidity adjusting materials, which are exactly same two samples chosen for the noiseabsorption process. This conclusion provides the likelihood to combine openpore and close-pore perlite to prepare a new integrated coating sample.

\subsection{Noise absorption coefficient test of integrated expanded-perlite sample.}

A new integrated expanded-perlite sample, named sample 5\#, combines openpore perlite, close-pore perlite, and a few woody fibres. Compared with expanded-perlite, woody fibre has a smaller porous diameter which can cause a good performance in noise absorption at a low-frequency. The method and condition of this experiment is the same process with 2.1.1. The coating has woody fibre of $1 \%-5 \%$, open-pore expanded perlite $15 \%-25 \%$, and close-cell expanded perlite $5 \%-15 \%$. After the same experiment process, the result is shown in the following Fig. 3.

Fig. 3 shows a great improvement of noise absorbing performance in lowfrequency. According to a former study, expanded-perlite has good absorbing ability in high-frequency which means that woody fibre improves inside porous structure. Along with an increase of noise frequency, the integrated coating sample can reduce noise better. As discussed above, people are usually sensitive of high-frequency noise. So, this integrated coating sample is very close to the actual application. 


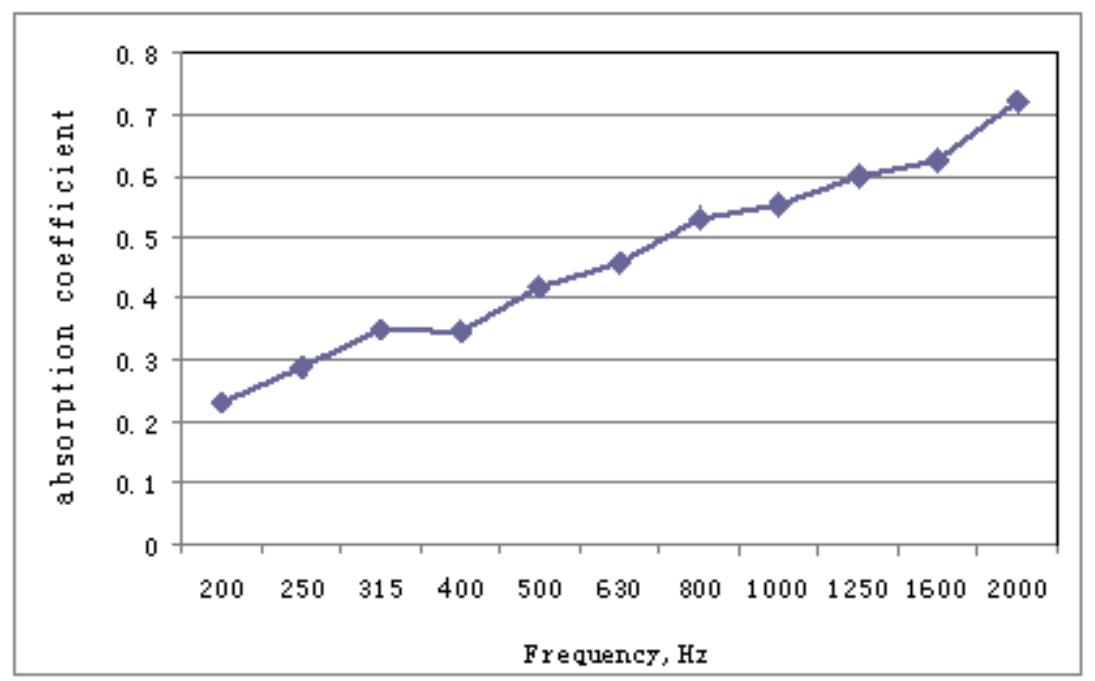

Figure 3: Noise absorption coefficient of integrated coating sample.

\section{Principle of noise absorbing}

According to Huygens' Principle, porous noise-absorbing material, with its open pore structure, has a certain character of aeration. When the noise waves impact upon the surface of porous materials, there are two main causes which lead to noise reduction. Firstly, the vibration, caused by noise waves, results in air movement inside pores and spaces. Therefore, friction happens between air molecules and the pore wall. However, air molecules closed to the pore wall and surface fibre are hard to move optionally. With the effect of friction and viscous force, a considerable portion of noise energy turns into thermal energy which causes a reduction of noise waves and reflection to achieve the purpose of noise absorption. Secondly, air flows within pores leads to a thermal exchange between the pore wall and surface fibre, which is also a reason of reduction of thermal energy.

The problem is that multi-porous material usually has a good performance of noise absorption in high-frequencies because high-frequency noise waves speed up vibration of air particles in interspaces and thermal exchange as well. In order to improve the noise absorbing performance in low-frequencies, woody fibre without poison, smell, pollution, and radioactivity was chosen as the new additive.

Woody fibre builds a 3D network within the integrated coating and improves viscosity of dried paint. It solves the problem of low durability caused by brittle structure of dry expanded-perlite coating. Expanded-perlite particles have extensive inner pore channels; and the original coating sample also has lots of inside cavities. As an important additive, woody fibre becomes the media to 
interconnect these pores and cavities. Therefore, this new integrated coating sample has better noise absorption coefficient than before.

\section{Findings and discussion}

As seen in these figures and tables, in terms of noise absorption coefficient the performance at medium and high frequency is better than at low frequency. Through noise absorption experiments, it is proved that open and close pore perlite has a good performance at some frequencies. It is also found that closedpore expanded perlite has a good quality at high frequency. And, in a humidity adjusting experiment, it is found that open and close pore perlite is the better of two kinds.

In order to improve the noise absorption at low frequency, one kind of woody fibre is designed as an additive to the coating. After addition of the woody fibre, the performance of noise-absorption in low frequencies has been improved relatively; but it still needs to be further improved.

\section{Conclusions}

A novel integrated noise absorbing coating has been designed without causing any organic pollution. It could be easily sprayed like normal water-based coating, and also has artistic effect because of its natural stereoscopic decorative effect. According to mature coating technology and expanded-perlite processing technology, productive cost can be controlled within an acceptable level by normal consumers. The most important characteristic of this material is that it also combines a humidity controlling ability which allows relative future product to adapt to any environments of the world.

\section{Acknowledgements}

This work has been made with the financial support of a grant from the National Key Technology R\&D Program in the 11th Five-year Plan of China, 2006BAJ02B02.

The authors are grateful to the support from the Institute of Acoustic, Chinese Academy of Sciences.

\section{References}

[1] Zhou Hong, Li Bo, Huang Guangsu, Sound absorption behavior of multiporous hollow polymer micro-spheres, Materials Letters, 60,pp.3451$3456,2006$.

[2] C.Zwikker, C.W.Kosten, Sound Absorption Materials, Elsevie.1949.

[3] H.Zhou, G.S.Huang, X.R.Chen, et al., Advances in sound absorption polymer, Progress in Chemisty, 16(3), 2004. 
[4] H.Zhou, B.Li. G.S. Huang, Sound absorption characteristics of polymer micro-particles, Polymeric Materials Science and Engineering, 20(3), 2004.

[5] J.Hbelt, A.Zeibig, C.Kostmann, G.Stephani, Parameter of metallic hollow spheres - a porous sound absorbing material, Proceedings of ICA2004, $18^{\text {th }}$ International Congress on Acoustics,2004, Kyoto, Japan.

[6] Krishan K. Ahuja, United States Patent, 5,777,947.

[7] Xu Chuanyou, Wang Haijuan, The Effect of Pearlite on Porous Sound Absorption Material, China non-metallic minerals industry,5,pp.2729,2008 .

[8] S.Meiarashi, M.Ishida, Noise Reduction Characteristics of Porous Elastic Road Surface. S. Meiarashi et al.pp.239-250, 1995.

[9] Semiha Yilmiha, Mesut B. Ozdeniz, The effect of moisture content on sound absorption of expanded perlite plates, Building and Environment, 40, pp.311-318, 20054.

[10] Wolfe R W, Gjinolli A. Durability and strength of cement-bonded wood particle composites made from construction waste, Forest Prod J, 49(2), pp.24-31, 1999.

[11] Harris DA. Noise control manual, New York: Van Nostrand Reinhold, pp.9-21, 1991. 